\title{
Optical Bumps in Cosmological GRBs as Supernovae
}

\author{
J.S. Bloom ${ }^{1,2}$
}

1 Harvard-Smithsonian Center for Astrophysics, MC 20, 60 Garden Street, Cambridge, MA 02138, USA; jbloomQcfa.harvard.edu

2 Harvard Society of Fellows, 78 Mount Auburn Street, Cambridge, MA 02138, USA

Summary. From both photometric and broadband spectral monitoring of $\gamma$-ray burst (GRB) lightcurve "bumps," particularly in GRB011121, a strong case grew for a supernova (SN) origin. The GRB-SN connection was finally solidified beyond a. reasonable doubt with the discovery that the bump in GRB 030329 was spectroscopically similar to a bright Type Ic SN. In light of this result, I re-assess the previous SN bump claims and conclude that: 1) the distribution of GRB-SN bump peak magnitudes is consistent with the local Type Ib/c SNe peak distribution and 2) the late-time bumps in all long-duration GRBs are likely supernovae.

\section{Introduction}

The connection of GRBs to supernovae (SNe) - and hence the death of massive stars - was explored theoretically [7] even before the discovery of GRBs. In the subsequent discovery paper, Klebesadel, Strong, \& Olson [15] reported on a catalog search for SNe coincident in time and position with the first 13 GRBs known. In retrospect, the lack of a clear association is easy to explain: not only were SNe sparsely discovered, but the highest redshift SN discovered by 1973 (SN 1968P; $z=0.11$ ) was less than the redshift of all $\sim 35$ classic GRBs redshifts known today. With the development of a model for the production of a GRB from a collapsing massive star [23], came renewed interest in the possibility of a GRB-supernova connection. The modern form of, and new spins on, Woosley's collapsar was presented in great detail by Andrew MacFadyen (this meeting).

The discovery of the low redshift SN 1988bw, a bright type Ic associated with GRB 980425 [9] implied that massive stars were capable of producing at least some incarnation of a GRB; the low energy release of GRB 980425 compared with all other known cosmological GRB energies suggests it may be only a sub-class of GRBs [1]. Attempts to theoretically unify GRB 980425 with the majority of the long-duration cosmological $(z>0.1)$ bursts fall short of explaining all the observational differences (see papers by Iwamoto and by Patat at this meeting).

Our discovery of a faint red light curve "bump" superimposed on the afterglow of GRB 980326 [2] was the first suggestion of a SN associated with 
a cosmological GRB. Supernovae-like features were later found photometrically in a number of bursts, some more plausible than others; the principal difficulty is that $\mathrm{SNe}$ peak at a brightnesses comparable to their respective host galaxies (see Fig. 1; $[4,5]$ ). Our multicycle effort on HST was designed to search for late-time afterglow features and discriminate between other physical models for such emission. While a few bursts showed no evidence for a SN (e.g., [20]), the late-time multicolor HST light curve and broadband spectra of GRB 011121 was reliably modeled by a bright Type Ic supernova that occurred nearly contemporaneously with the GRB [3]. Moreover, this was the first burst for which the data were of high enough quality to statistically demonstrate that the afterglow propagated in a wind-stratified medium rather than a constant-density ISM [19]. I recently reviewed the pre-2003 evidence for the GRB-SN connection in [5].

\section{GRB 030329 and Beyond}

While the case was rather strong for late-time emission bumps as being due to underlying supernovae [5], the definitive direct spectroscopic evidence was found only recently with the low-redshift ( $z=0.17$; [11]) GRB 030329. For this burst, a number of groups $[8,12,14,22]$ found a bump with supernova absorption features indicative of very high velocity ejecta. The close evolutionary track of this apparent supernova (2003dh; [10]) with SN 1998bw should quell any remaining doubt that long-duration GRBs are produced in the death of massive stars.

The rare proximity of this event allowed for high-precision coverage from the ground around the SN peak (e.g., $[16,17]$ ). Using optical/IR imaging at the CTIO $1.3 \mathrm{~m}$ to infer the line-of-sight dust extinction, we inferred that the SN was roughly 0.3 mag brighter at peak than the bright Type Ic 1998bw [6]. Since the precise peak SN magnitude depends on the line of-sight extinction toward the GRB, it is imperative to infer extinction from the early afterglow in future events. With new dedicated robotic IR systems and space-based UV imaging, the broadband spectral coverage (from 0.1 to $2 \mu \mathrm{m}$ ) of Swift afterglows should routinely be used to infer line-of-sight extinction.

The peak brightness and early evolution of SN 2003dh, when compared to $1998 \mathrm{bw}$, enabled estimates of the total mass of the ejecta $\left(8 \mathrm{M}_{\odot}\right)$, synthesized ${ }^{56} \mathrm{Ni}$ mass $\left(0.35 \mathrm{M}_{\odot}\right)$, and mass of the progenitor $\left(\sim 35 \mathrm{M}_{\odot}\right)$ [18]. At this meeting, I suggested that a great deal more could be learned with a concerted long-term effort on HST to resolve and follow the evolution of the SN as it faded below the integrated brightness of the host galaxy. A measurement of the polarization of the light curve during the decay phase would constrain the asphericity of the emission region $[13,24]$. If the late-time evolution of SN $2003 \mathrm{dh}$ was indeed powered by radioactive decay of ${ }^{56} \mathrm{Co}$ to ${ }^{56} \mathrm{Fe}$, then the time history of the integrated luminosity would also determine the amount of synthesized ${ }^{56} \mathrm{Ni}$ (now only inferred by scaling $1998 \mathrm{bw}$ ). To be sure, leakage 


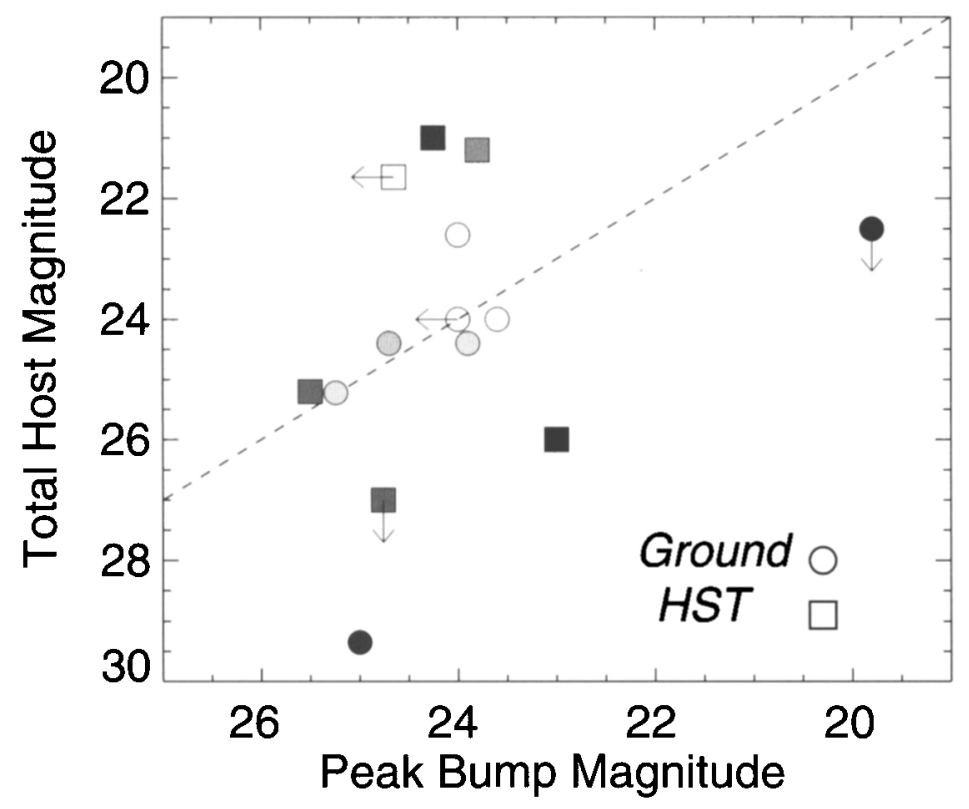

Relative Bump Significance
High

Fig. 1. The difficulty of detecting supernovae associated with GRBs: the magnitude of the host galaxy versus the peak magnitude of the putative bump (in whichever optical filter the peak bump magnitude was reported). Above the dashed line, bump magnitudes are fainter than the integrated light of the host galaxy. The circles are measurements where only ground-based imaging was used and squares are measurements derived also using Hubble Space Telescope (HST) imaging. Relative significances of the reliability of the bump detection were assigned by the author based roughly on the number of detections of the bump above the host light and the photometric error reported on the bump peak. Updated (from [5]) with new bumps in GRB 030329 and GRB 021211, reported at this meeting.

of $\gamma$-rays produced by radioactivity from the expanding nebula would make the late-time decay steeper than a pure ${ }^{56} \mathrm{Co}$ exponential.

Though some of the details of SN 2003dh have not been flushed out observationally, it seems clear that a bright core-collapse supernova and GRB 030329 occurred nearly simultaneously (to less than 1 week) $[16,17]$. A pressing question is now whether a supernova accompanies all long-duration GRBs. On this point, a brief caution: while bumps have been claimed in more than 10 events, a number of these may be spurious due to improper subtraction of the host light. Fig. 1 shows a compilation of the peak brightnesses of all late-time bumps reported to-date in comparison with the total magnitude of the respective host galaxy. Five bumps have been found to peak within 


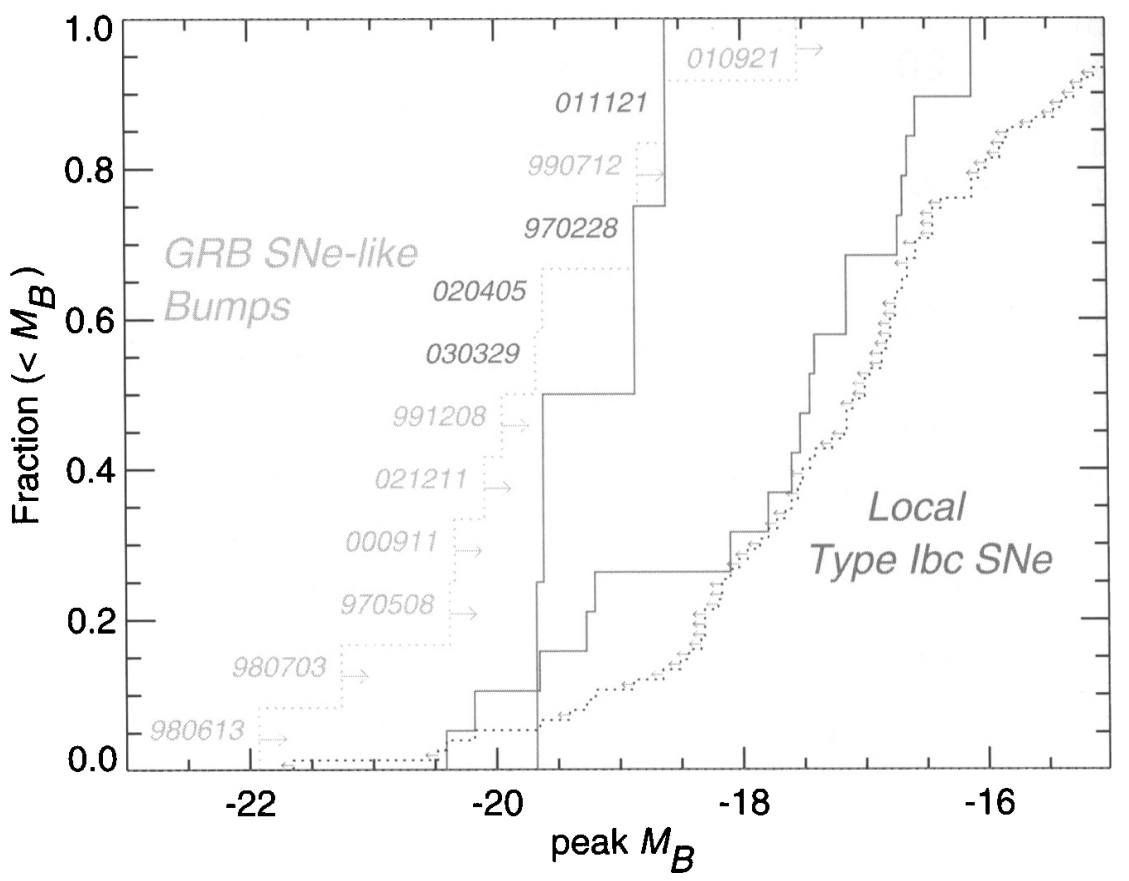

Fig. 2. Comparison of the peak magnitudes of GRB-SNe with those of local Type Ib/Ic SNe. The solid cumulative histogram to the left is for those GRBs with a believable detection of a SN bump; the brightnesses of all other claimed GRB-SN peaks or reported upper-limits are shown as a dotted histogram. The solid histogram to the right is for those local $\mathrm{Ib} / \mathrm{c}$ SNe for which the SN was observed before peak; all others were discovered after peak. The faintest GRB-SN non-detection (GRB 010921) only probes the top 40th-percentile of local Type Ib/Ic SNe. It is clear that current GRB-SNe population may have only revealed the tip of the iceberg; plausibly, then, SNe could accompany all long-duration GRBs. Compilation of local $\mathrm{Ib} / \mathrm{c}$ SNe from [21].

0.3 magnitudes of hosts at $\sim 24-26$ mag, uncomfortably close for photometric differencing at such faint levels.

Of the bursts with redshift, I believe that only GRBs 030329, 020405 , 970228 , and 011121 can be legitimately claimed as true detections of SNlike bumps related to a GRB. This statement is not intended to imply that SNe do not accompany long-duration GRBs. Instead, the SN peaks may simply have fallen below the detection threshold of the instrument and/or the brightness of the host galaxy. How do these non-detections compare with the expected brightnesses of GRB-SNe? Even if all GRB-SNe are of type $\mathrm{Ib} / \mathrm{c}$, it is important to note that there is no a priori theoretical reason to require that all GRB-SNe should be identical: observed local Type Ib/c SNe show rather diverse light curves, peak brightnesses and spectral evolution. 
Thus, the simplest assumption is that the peak magnitude distribution of GRB-SNe should follow the distribution of local Type Ib/c SNe.

Figure 2 shows a comparison of the distribution of absolute $B$-band peak magnitudes GRB-SNe (with known redshift) and local Ib/c SNe. After correcting for the extinction due to dust in our Galaxy, I have (simplistically) $k$-corrected the observed (or lower-limit) peak magnitude to the rest frame $B$ band using the observed redshift and assuming a blackbody spectrum peaked in the rest frame $V$-band. The uncertainty introduced by this approximation on an individual $M_{B}$ is likely less than $0.5 \mathrm{mag}$. From the figure, it is clear that most GRB-SNe detections (or limits) only probe the bright end of the local $\mathrm{Ib} / \mathrm{c}$ population. In fact, the deepest non-detection of a GRB-SN (GRB 010921) only probes the brightest 40th percentile of local $\mathrm{Ib} / \mathrm{c}$ SNe. From Fig. 2, it is reasonable to conclude that the observed supernovae related to GRBs have only probed the tip of the iceberg of a plausible brightness distribution. A systematic survey of late-time bumps from nearby $(z \lesssim 0.5)$ Swift bursts will test this hypothesis.

\section{References}

1. J.S. Bloom et al. : Astrophys. J. Lett. 506, L105 (1998)

2. J.S. Bloom et al. : Nature 401, 453 (1999)

3. J.S. Bloom et al. : Astrophys. J. Lett. 572, L45 (2002)

4. J.S. Bloom: astro-ph 0301028

5. J.S. Bloom: astro-ph 0303478

6. J.S. Bloom et al. : Astron. J. 127, 252 (2004)

7. S.A. Colgate: Canadian J. Phys. 46, 476 (1968)

8. R. Chornock et al. : IAUC 8114 (2003)

9. T.J. Galama et al. : Nature 395, 670 (1998)

10. P. Garnavich, T. Matheson, E.W. Olszewski, P. Harding, K.Z. Stanek: IAUC 8114 (2003)

11. J. Greiner et al. : GCN 2020

12. J. Hjorth et al. : Nature 423, 847 (2003)

13. K. Iwamoto et al. : Nature 395, 672 (1998)

14. K.S. Kawabata et al. : Astrophys. J. Lett. 593, L19 (2003)

15. R.W. Klebesadel, I.B. Strong, R.A. Olson: Astrophys. J. Lett. 182, L85 (1973)

16. Y.M. Lipkin et al. : in preparation

17. T. Matheson et al. : in press

18. P.A. Mazzali et al. : astro-ph 0309555

19. P.A. Price et al. : Astrophys. J. Lett. 572, L51 (2002)

20. P.A. Price et al. : Astrophys. J. 584, 931 (2003)

21. D. Richardson et al. : Astron. J. 123, 745 (2002)

22. K.Z. Stanek et al. : Astrophys. J. Lett. 591, L17 (2003)

23. S.E. Woosley: Astrophys. J. 405, 273 (1993)

24. S.E. Woosley, R.G. Eastman, B.P. Schmidt: Astrophys. J. 516, 788 (1999) 